\title{
Synthesis of Stably Water-Soluble Gold Nanoparticles Protected by Porphyrin-Thiol Derivative*
}

\author{
Masayuki Kanehara and Toshiharu Teranishi ${ }^{\dagger}$ \\ Graduate School of Pure and Applied Sciences, University of Tsukuba, \\ 1-1-1 Tennodai, Tsukuba, Ibaraki 305-8571, Japan
}

(Received 28 December 2004; Accepted 19 January 2005; Published 3 February 2005)

\begin{abstract}
Meso-tetraphenylporphyrin derivative having four thioacetyl groups was synthesized using Lindsay's method. Monodisperse small gold $(\mathrm{Au})$ nanoparticles passivated by the synthesized ligand were prepared by a liquid phase synthesis. The nanoparticles were extremely stable and can be homogeneously dispersed in basic aqueous solution. [DOI: $10.1380 /$ ejssnt.2005.30]
\end{abstract}

Keywords: Gold; Nano-particles, quantum dots, and supra-molecules; Porphyrin; Thiol

\section{INTRODUCTION}

Metal nanoparticles play important roles in different branches of science, such as nanoelectoronics, nonlinear optics, biological labeling, oxidation catalyst, etc. Nanoparticles themselves also provide a pragmatic approach to the multiscale engineering, functioning as 'building blocks' of regular shape and size for the fabrication of lager structures. Combination of synthetic design with directed assembly of nanoparticles into ensembles provides direct control of structure from the molecular to the macroscopic level. In this regard, functionalization of metal nanoparticles is a prerequisite to fabrication 2- and 3-dimensional structures. Though a lot of reports on terminal functionalization of the ligands protecting metal nanoparticles have been reported based on both direct syntheses and ligand exchange reactions [1-9], thiol-terminated nanoparticles are still rare. Unique and high-selective reactivities of thiol groups may open the new door for the fabrication of macroscopic structures. Here we report the synthesis of newly-designed tetradentate thiol ligand, meso-tetra(3mercaptophenyl)porphyrin (TMP), and the preparation of monodisperse Au nanoparticles protected by the TMP ligand.

\section{EXPERIMENTAL}

The synthetic procedure of TMP ligand is as follows (see Fig. 1): $m$-Toluenethiol was S-acetylated using acetic anhydride to afford thioester $\mathbf{1}$ as colorless oil in $97 \%$ yield, and the subsequent standard NBS bromination in benzene gave benzylbromide $\mathbf{2}$ as pale yellow oil in $82 \%$ yield. DMSO oxidation of $\mathbf{2}$ at room temperature provided aldehyde $\mathbf{3}$ as pale yellow oil in moderate yield, 44\%. TMP ligand was obtained using Lindsay's method [10] as a purple solid in $41 \%$ yield. All compounds were identified by ${ }^{1} \mathrm{H}-\mathrm{NMR}$ and EI- or ESI-MS.

The TMP-protected Au (TMP-Au) nanoparticles were synthesized by the chemical reduction of $\mathrm{Au}(\mathrm{III})$ to $\mathrm{Au}(0)$ with $\mathrm{NaBH}_{4}$ as a reducing agent in the presence of one

\footnotetext{
* This paper was presented at International Symposium on Nanoorganization and Function, Tokyo, Japan, 11-12 November, 2004 †Corresponding author: teranisi@chem.tsukuba.ac.jp
}

equivalent of ligands to Au species [11]. In a typical preparation, $10 \mu \mathrm{mol}$ of $\mathrm{HAuCl}_{4} \cdot 4 \mathrm{H}_{2} \mathrm{O}$ was dissolved in $45 \mathrm{~mL}$ of $N, N$-dimethylacetamide (DMAc) containing $10 \mu \mathrm{mol}$ of TMP ligand, and then $100 \mu \mathrm{mol}$ of $\mathrm{NaBH}_{4}$ in $5 \mathrm{~mL}$ of methanol was swiftly added under vigorous stirring. After 12 hour stirring at room temperature, the solvent was completely evaporated and the resulting precipitate was dissolved in water and purified using Sephadex G-25 to give pure TMP-Au nanoparticle aqueous solution.

\section{RESULTS AND DISCUSSION}

On adding the reducing agent into the DMAc solution containing $\mathrm{HAuCl}_{4} \cdot 4 \mathrm{H}_{2} \mathrm{O}$ and TMP, the color of the solution turned from red-purple to dark-red immediately, indicating the formation of the $\mathrm{Au}$ nanoparticles. After the solvent evaporation, the TMP-Au nanoparticles were purified by Sephadex G-25. The purified aqueous TMP-Au nanoparticles dissolved in water were extremely stable under an ambient condition. Even after 1 month, no precipitates were formed, and the color of the solution remained unchanged. This result surprised us, because the water soluble TMP-Au nanoparticles are expected to have free thiolate groups, which are readily oxidized to form water-insoluble disulfide under an ambient condition. Presumably, an oxidation rate of the free thiolates is too slow in our system due to steric hindrance. The TMP ligands coordinated on the Au nanoparticle surfaces possess a micelle-like structure, and such a crowded environment might suppress the formation of disulfides.

Figure 2 shows the UV-vis spectra of the DMAc solution containing TMP and Au ion (solid line) and purified aqueous solution of TMP-Au nanoparticles (dotted line). After the reduction of $\mathrm{Au}$ ion, the surface plasmon resonance band around $520 \mathrm{~nm}$ has appeared, indicating the formation of $\mathrm{Au}$ nanoparticles. The Soret band of porphyrin rings at $420 \mathrm{~nm}$ on the gold surface was broadened, indicating the formation of stacking structure of porphyrin ring. Similar broadening of the Soret band was reported for porphyrin SAMs on gold electrodes [12], LB monolayers of porphyrin [13], and porphyrin aggregates in solutions [14].

The TMP-Au nanoparticles were placed onto a formvarcoated $\mathrm{Cu}$ grid with a small amount of ethylene glycol (ca. $1 \%$ volume to the solution) for TEM observation. Ethylene glycol improves the affinity of the solvent toward the formvar membrane during the solvent evaporation, and 
<smiles>Cc1cccc(S)c1</smiles>

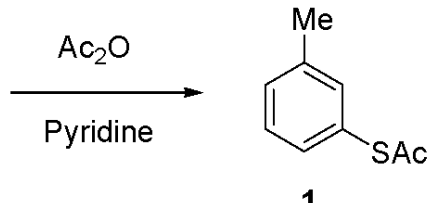

$97 \%$<smiles>O=Cc1cccc(S(=O)(=O)c2ccccc2)c1</smiles><smiles>c1cc[nH]c1</smiles>

1, $\mathrm{CF}_{3} \mathrm{COOH}, \mathrm{BF}_{3} / \mathrm{CH}_{2} \mathrm{Cl}_{2}$ 2. Chloranil, $\mathrm{NEt}_{3} / \mathrm{CH}_{2} \mathrm{Cl}_{2}$

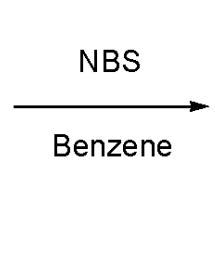

$82 \%$

2

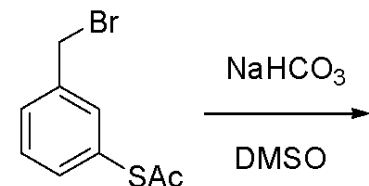

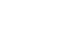

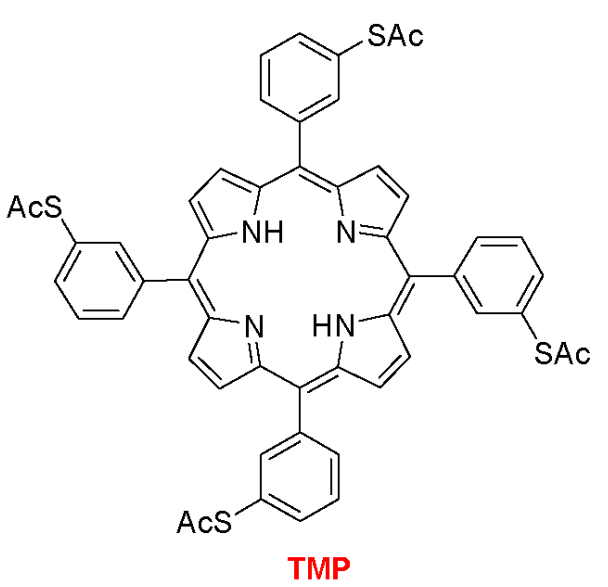

$41 \%$<smiles>O=Cc1cccc(S(=O)(=O)[O-])c1</smiles>

$44 \%$

FIG. 1: The synthetic procedure of TMP ligand.

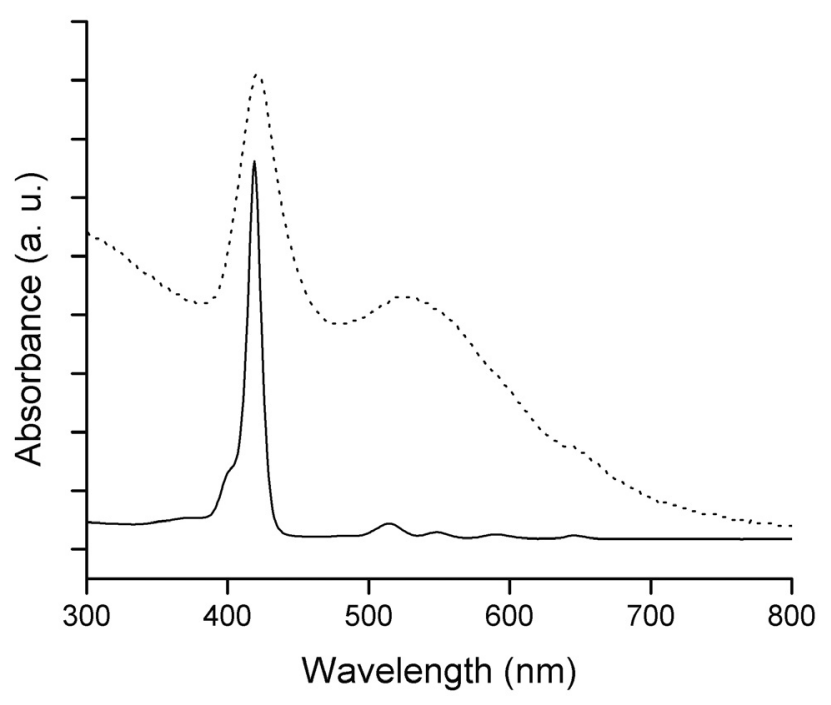

FIG. 2: UV-vis spectra of before reduced DMAc solution containing TMP ligand and $\mathrm{HAuCl}_{4}$ (solid line) and purified aqueous TMP-Au nanoparticle solution (doted line).

helps the formation of superlattices of Au nanoparticles [7]. Figure 3 shows the TEM image and size distribution histogram of the TMP-Au nanoparticles. The TMP$\mathrm{Au}$ nanoparticles have small diameter with very narrow size distribution, $2.3 \pm 0.2 \mathrm{~nm}$. Generally, strong reducing agent and ligand with strong coordination ability, which serve for fast nucleation and strong protection, respectively, are necessary to prepare small nanoparticles [15]. In our case, $\mathrm{NaBH}_{4}$ is strong enough to make small $\mathrm{Au}$ nanoparticles, although the TMP ligand has weak coordinative thioacetyl groups. Tetradentate TMP ligand with four thioacetyl groups may contribute the strong protection to the $\mathrm{Au}$ nanoparticles. Once the TMP ligands protect the $\mathrm{Au}$ surfaces, thioester groups spontaneously dissociate to form thiolates and generate self-assembled monolayer on bare Au surface [10]. Consequently, the highly stably water-soluble TMP-Au nanoparticles were obtained.

\section{CONCLUSIONS}

In conclusion, newly-synthesized tetradentate thioester ligand, TMP, has synthesized. Monodisperse small TMPprotected $\mathrm{Au}$ nanoparticles were prepared by the liquid phase reduction of $\mathrm{Au}$ ion in the presence of the TMP ligand. The water-soluble TMP-Au nanoparticles were extremely stable under an ambient condition, and steric hindrance around thiolate groups may contribute the stability against the disulfide formation. The TMP-Au nanoparticles have a potential application for the building blocks by exploiting the selective reactivity of terminal thiolate groups.

\section{Acknowledgments}

This work was supported by Grant-in-Aid for Young Scientists (A) (No.15681009) from the Ministry of Education, Culture, Sports, Science and Technology, Japan (T.T.). 

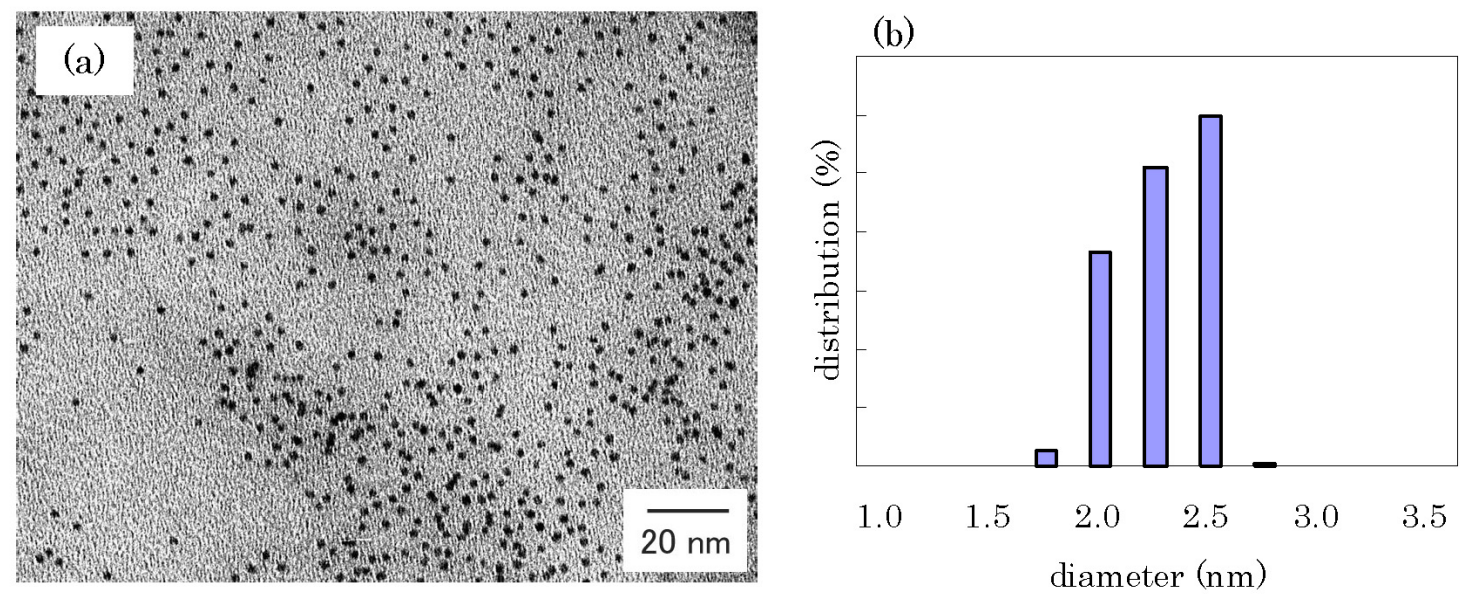

FIG. 3: (a) Representative TEM image and (b) size distribution histogram of TMP-Au nanoparticles.

[1] A. K. Boal, F. Ilhan, J. E. Derouchey, T. Thurn-Albrecht, T. P. Russell and V. M. Rotello, Nature 404, 746 (2000).

[2] T. H. Galow, A. K. Boal and V. M. Rotello, Adv. Mater. 12, 576 (2000).

[3] B. L. Frankamp, A. K. Boal and V. M. Rotello, J. Am. Chem. Soc. 124, 15146 (2002).

[4] R. Shenhar and V. M. Rotello, Acc. Chem. Res. 36, 549 (2003).

[5] M. M. Maye, S. C. Chun, L. Han, D. Rabinovich and C.-J. Zhong, J. Am. Chem. Soc. 124, 4958 (2002).

[6] M. J. Hostetler, S. J. Green, J. J. Stokes and R. W. Murray, J. Am. Chem. Soc. 118, 4212 (1996).

[7] M. Kanehara, Y. Oumi, T. Sano and T. Teranishi, J. Am. Chem. Soc. 125, 8708 (2003).

[8] M. Kanehara, Y. Oumi, T. Sano and T. Teranishi, Bull. Chem. Soc. Jpn. 77, 1589 (2004).

[9] G. Schmid, M. Baumle and N. Beyer, Angew. Chem. Int.
Ed. 39, 181 (2000)

[10] D. T. Gryko, C. Clausen, K. M. Roth, N. Dontha, D. F. Bocian, W. G. Kuhr and J. S. Lindsey, J. Org. Chem. 65, 7345 (2000).

[11] M. Brust, M. Walker, D. Bethell, D. J. Schiffrin and R. Whyman, Chem. Commun. 1994, 801 (1994).

[12] J. Zak, H. Yuan, M. Ho, L. K. Woo and M. D. Porter, Langmuir 9, 2772 (1993).

[13] D. Gust, T. A. Moore, A. L. Moore, D. K. Luttrull, J. M. Degraziano, N. J. Boldt, M. Van Der Auweraer and F. C. De Schryver, Langmuir 7, 1483 (1991).

[14] N. C. Maiti, S. Mazumdar and N. Periasamy, J. Phys. Chem. B 102, 841 (1998).

[15] A. C. Templeton, W. P. Wuelfing and R. W. Murray, Acc. Chem. Res. 33, 27 (2000). 